\title{
Elternzeit - Teilzeit - Aus(zeit)? Teilzeitrechte in Führungspositionen
}

Seit 2001 gelten in der Bundesrepublik erstmals Teilzeitrechte, die Beschäftigten neue Handlungsoptionen in Phasen der Kinderbetreuung ermöglichen sollen. Ausdrücklich fördern wollte der Gesetzgeber reduzierte Arbeitszeiten auch in den Führungsetagen von Deutschlands Unternehmen und verband damit das Ziel, die Gleichstellung von Frauen und Männern zu verbessern. Die Arbeitgeberverbände lehnten vor der Verabschiedung im Parlament die neuen Rechte grundsätzlich ab. Im Mittelpunkt des Beitrags stehen empirische Forschungsergebnisse zu der Frage, wo Barrieren im Umgang mit den Rechtsansprüchen in der betrieblichen Praxis bei Führungskräften mit Kindern bestehen und welche Potenziale die neuen Normen bieten.

\section{Einleitung}

Wie lassen sich in qualifizierten beruflichen Positionen Berufstätigkeit und Familie miteinander kombinieren? Wie erklärt sich, dass - trotz Bildungsexpansion und gesetzlichen Gleichstellungsfortschritten - die vertikale Geschlechtersegregation auf dem Arbeitsmarkt so ausgeprägt ist und in Führungspositionen in Deutschland noch immer vergleichsweise selten Frauen und erst recht nicht Mütter kleiner Kinder zu finden sind? Diese Fragen sind Teil einer gesellschafts-, arbeits- und geschlechterpolitischen Debatte, die in der wissenschaftlichen und politischen Diskussion der letzten beiden Jahrzehnte eine zentrale Bedeutung hatte. Die zwar altbekannte, jedoch keineswegs gelöste Problematik hatte durch die arbeits- und sozialpolitischen Reformgesetze, die die rot-grüne Bundesregierung im Jahr 2001 verabschiedete, besondere Aktualität erfahren: Mit dem Bundeserziehungsgeldgesetz und dem Teilzeit- und Befristungsgesetz erhielten Beschäftigte mit Kindern über Rechtsansprüche auf Arbeitszeitreduzierung in und nach der Elternzeit bessere Möglichkeiten, Berufs- und Fürsorgearbeit miteinander zu vereinbaren. Damit wurde die wohlfahrtstaatliche Regulierung im Hinblick auf die arbeitszeitpolitische Komponente der Vereinbarkeit von Erwerbstätigkeit und Familie grundlegend verändert. Im internationalen Vergleich repräsentierte die Bundesrepublik bis dato über Jahrzehnte hinweg ein Modell familienorientierter Sozialpolitik, das die Diskontinuität des Erwerbsverlaufs und eine ausschließliche Betreuung der Kinder durch die Mutter förderte (Klammer/Daly 2003, S. 203; Koch 2001, S. 50f.). Als Konsequenz für die Arbeitsmarktpartizipation sind nach wie vor unterschiedliche Beschäftigungschancen, Erwerbsmuster und Berufsverläufe von Frauen und Männern festzustellen, die zu geschlechterspezifischen Ungleichheiten am Arbeitsmarkt führen.

Kennzeichnend für die vertikale Geschlechtersegregation auf den höchsten Ebenen der betrieblichen Hierarchie ist, dass nach Ergebnissen des Mikrozensus weniger als ein Viertel (23\%) aller Führungspositionen in der Privatwirtschaft im Jahr 2004 mit Frauen besetzt war (Kleinert et al. 2007, S. 62). Vollzeitarbeitsverhältnisse dominieren: Von den abhängig beschäftigten Frauen in Führungspositionen in der Privatwirtschaft arbeiteten im Jahr 2004 $14 \%$ in Teilzeit, bei den männlichen Führungskräften waren es $2 \%$ (ebd., S. 81f.). Auch die überlangen Arbeitszeiten von Beschäftigten in Führungspositionen lassen eine parallele Kombination von Erwerbstätigkeit und Betreuungsarbeit bei hoch qualifizierter Beschäftigung als kaum möglich erscheinen. ${ }^{1}$

Wie gehen Personalverantwortliche mit den neuen Arbeitszeitrechten für den Führungskräftebereich um, dem Segment, in dem entgrenzte Arbeitszeiten zur betrieblichen Normalität gehören? Wo liegen Barrieren, wo Potenziale der neuen sozialen Rechte in der betrieblichen Praxis? In diesem Beitrag werden empirische Forschungsergebnisse vorgestellt, wie sich der Umgang mit den gesetzlichen Teilzeitrechten bei Führungskräften mit Kindern während und nach der Elternzeit durch die Arbeitgeberseite gestaltet. ${ }^{2}$ Dabei liegt der Fokus auf zwei Analyseschwerpunkten:
Zunächst wird gezeigt, welche Argumentationsmuster im Umgang mit den Teilzeitansprüchen vorherrschen. Beleuchtet wird hier, welche Kluft zwischen den gesetzlichen Vorgaben und den Perspektiven derjenigen besteht, die diese umsetzen sollen (Abschnitt 3). Im zweiten Schwerpunkt wird anhand der Ergebnisse von Fallanalysen der Frage nachgegangen, wie sich der restriktive betriebliche Umgang mit den Teilzeitrechten erklärt. Die Ursachen zeigen sich sowohl auf der Betriebsebene als auch in der individuellen Lebensführung, in Familienmodellen und Geschlechterkonstruktionen (Abschnitt 4). Der Analyse vorangestellt ist ein Blick auf bestehende Arbeitszeitrechte (Abschnitt 2). Abschnitt 5 zieht ein Fazit.

\footnotetext{
1 Nach Ergebnissen des Sozioökonomischen Panels (SOEP) arbeiteten vollzeitbeschäftigte Führungskräfte 2004 durchschnittlich 52 Stunden wöchentlich (Holst 2006, S. 366)

2 Im Rahmen einer qualitativen Untersuchung wurden 20 Personalverantwortliche des mittleren und oberen Managements aus fünf Großbetrieben unterschiedlicher Branchen in mehrstündigen Interviews befragt, darunter zwölf Männer und acht Frauen. Die hier vorgestellten Ergebnisse basieren auf einem 2007 abgeschlossenen und von der Hans-Böckler-Stiftung geförderten Forschungsprojekt zur Evaluation der Neuregelungen des Bundeserziehungsgeldgesetzes und des Teilzeitund Befristungsgesetzes (Bäcker et al. 2007).
}

Angelika Koch, Dr., Vertretungsprofessur am Institut für Politikwissenschaft, Universität Duisburg-Essen. Arbeitsschwerpunkte: Arbeits- und Sozialpolitikforschung, Gender Studies, Qualitative Methoden. e-mail: angelika.koch@uni-due.de 


\section{Stärkung der Arbeitszeitrechte}

Mit der Verabschiedung des Bundeserziehungsgeldgesetzes (BErzGG 2001) und des Teilzeit- und Befristungsgesetzes (TzBfG 2001) wurde in Deutschland die Bindung von Beschäftigten mit Kindern an den Arbeitsmarkt erstmals über individuelle Arbeitnehmerrechte gestärkt. Damit wurden die gesetzlichen Möglichkeiten verbessert, Erwerbstätigkeit und Betreuungsarbeit in und nach der Elternzeit zu kombinieren. Im Kern setzte der Gesetzgeber Forderungen um, die seit Jahrzehnten in der wissenschaftlichen und politischen Diskussion zu der Frage, wie Erwerbstätigkeit und Familienarbeit zu vereinbaren sind und gesellschaftliche Arbeit verteilt werden kann, eine wichtige Rolle spielten. ${ }^{3}$ War Teilzeitarbeit bisher mit einem eingeschränkten Berufsspektrum und niedriger Entlohnung auf den unteren Ebenen der betrieblichen Hierarchie sowie mit zahlreichen rechtlichen Benachteiligungen verbunden, so sind die Rechtsansprüche ein wichtiger Schritt, sie als gleichberechtigte Arbeitszeitform anzuerkennen. ${ }^{4}$

Mit der Reform des Bundeserziehungsgeldgesetzes 2001 erhielten Beschäftigte in Elternzeit einen Rechtsanspruch, ihre Arbeitszeit auf ihrem Arbeitsplatz in einem Korridor von 15 bis 30 Stunden pro Woche $\mathrm{zu}$ reduzieren. Die schwachen Zeitrechte, die die Erwerbsbeteiligung von der $\mathrm{Zu}$ stimmung des Arbeitgebers abhängig machten und nach dem Muster des Zuverdienstes innerhalb der Versorgerehe gestalteten, wurden aufgehoben: Bis dato war die Option, Teilzeit bis maximal 19 Stunden wöchentlich in der Elternzeit zu arbeiten, in die Dispositionsfreiheit des Arbeitgebers gestellt.

Von ähnlicher Bedeutung für die Stärkung der Zeitrechte war, dass zum gleichen Zeitpunkt ein nicht auf bestimmte Lebensphasen bezogener Anspruch auf Arbeitszeitreduzierung durch das Teilzeit- und Befristungsgesetz eingeführt wurde. Beschäftigte mit Kindern haben seitdem das Recht, ihre Arbeitszeit auch im Anschluss an die Elternzeit zu reduzieren. In beiden Gesetzen wurden Ablehnungsgründe des Teilzeitanspruchs durch den Arbeitgeber normiert: Im Rahmen der Elternzeit kann der Arbeitgeber „dringende betriebliche Grün- $d e$ " geltend machen. Beim Rechtsanspruch nach dem Teilzeit- und Befristungsgesetz kann der Anspruch abgelehnt werden, wenn ihm „betriebliche Gründe“ entgegenstehen. Hierzu zählen laut Gesetz eine wesentliche Beeinträchtigung der Organisation, des Arbeitsablaufs oder der Sicherheit des Betriebs sowie unverhältnismäßige Kosten. Wichtig ist hierbei, dass der Gesetzgeber somit grundsätzlich Veränderungen im Arbeitsablauf, der Organisation des Betriebs wie auch Kosten von Arbeitszeitreduzierungen akzeptiert. ${ }^{5}$

Mit dem Teilzeit- und Befristungsgesetz wurde darüber hinaus eine Norm eingeführt, die in enger Verbindung mit der Teilzeitförderung durch den Rechtsanspruch auf Arbeitszeitreduzierung steht. Sie verpflichtet den Arbeitgeber, Stellen bei öffentlicher oder innerbetrieblicher Ausschreibung auch als Teilzeitarbeitsplätze auszuschreiben, wenn sich diese dazu eignen. Bereits durch Regelungen im Prozess des Stellenbesetzungsverfahrens kann damit dazu beigetragen werden, die Unterrepräsentanz von Frauen in hoch qualifizierten Positionen durch eine andere Rekrutierungspolitik abzubauen.

Betrachtet man die Einführung der Rechtsansprüche im Rahmen der Vereinbarkeitspolitik in Deutschland, so bieten sie eine wichtige Möglichkeit, Erwerbstätigkeit und Betreuungsarbeit flexibler zu gestalten. Aufgrund der noch immer geringen Betreuungsmöglichkeiten für Kinder unter drei Jahren wie auch für Schulkinder fehlen in Westdeutschland jedoch gleichzeitig wesentliche Rahmenbedingungen für ihre Umsetzung.

\section{Argumentationsmuster der Personalverantwortlichen}

Auf welche Haltungen trifft der Anspruch auf Teilzeit bei den befragten Personalverantwortlichen, wenn es um die Umsetzung im Führungskräftebereich geht? Zentrales Untersuchungsergebnis ist, dass die überwiegende Mehrheit der Personalverantwortlichen den Ansprüchen ablehnend gegenübersteht. Lediglich eine Personalverantwortliche in der gesamten Untersuchungsgruppe stand deren Umsetzung im Bereich von Führungspositionen positiv gegenüber. In der betrieblichen Praxis führt dies dazu, dass Arbeitszeitreduzie- rungen nur bei Verlust des betrieblichen Status erfolgen. Hierbei zeigen sich keine Differenzierungen im Hinblick auf Phasen in der Elternzeit oder nach der Elternzeit. Geschlechtspezifische Differenzierungen ließen sich ebenfalls nicht erkennen. Für zwei der untersuchten Unternehmen wurde deutlich, dass grundsätzlich keine reduzierten Arbeitszeiten in Leitungspositionen gewährt wurden.

Bezogen auf die Frage, ob Beschäftigte mit Kindern bei reduzierten Arbeitszeiten in Leitungsfunktionen beruflich aufsteigen können, lassen die Ergebnisse aus der Befragung der Personalverantwortlichen erkennen, dass bis auf zwei Ausnahmen keiner der Personalverantwortlichen höhere Positionen sowie Positionen mit Leitungsfunktionen mit reduzierter Arbeitszeit ausgeschrieben hat oder noch ausschreiben würde. ${ }^{6}$

\subsection{DAS POSTULAT ENTGRENZTER ARBEITSZEITEN}

Dominantes Argumentationsmuster für die ablehnende Haltung der Personalverantwortlichen gegenüber reduzierten Arbeitszeiten in Führungspositionen ist der Verweis auf einen entgrenzten Arbeitszeitstandard, der weit über Vollzeitarbeit hinausgeht. Über das Argumentationsmuster der uneingeschränkten Verfügbarkeit werden hoch qualifizierte Berufsarbeit und Sorgearbeit als unvereinbar konstruiert. Die Ausschließlichkeit der Bindung an die Berufsarbeit tritt im folgenden Interviewausschnitt besonders deutlich hervor.

3 Siehe hierzu Kurz-Scherf (1987); Bäcker/StolzWillig (1993); Pfarr (1994); zu den politischen Entwicklungen ausführlich Bothfeld (2005).

4 Zu den Benachteiligungen von Teilzeitarbeit und den Rechtsentwicklungen im Hinblick auf die Gleichbehandlung von Teilzeitbeschäftigten siehe Perreng/Nollert-Borasio (2006, S. 83ff.) sowie Buschmann et al. (2001, S. 329ff.). Zur Problematik von Teilzeitarbeit im Niedriglohnbereich siehe Weinkopf/Kalina (2006).

5 Ein weiterer Unterschied zwischen den beiden Ansprüchen besteht darin, dass das Recht auf Arbeitszeitreduzierung nach dem Teilzeit- und Befristungsgesetz nicht mit einer Rückkehroption auf die vorherige vertraglich vereinbarte Arbeitszeit verbunden ist.

6 Bei einer dieser Ausnahmen wurde jedoch in den weiteren Ausführungen zu einem konkreten Fall deutlich, dass die Arbeitszeitreduzierung zwar gewährt wurde, jedoch keine Reduzierung des Arbeitsumfangs erfolgte, sodass das Modell nach kurzer Zeit scheiterte. 
„Also ich kann keine Person in einer Führungsfunktion haben, von der ich nicht sicher bin, dass mir diese Person fünf Tage in der Woche je zwölf Stunden da ist" (Bereichsleiter Marketing).

Die Bereitschaft, uneingeschränkt zur Verfügung zu stehen, wird auch im Sinne höherer Leistungsorientierung gedeutet. Verfügbarkeit gilt unter dem Gesichtspunkt des Wettbewerbs als Konkurrenzmaßstab.

„Da ich natürlich in der Woche OpenEnd arbeiten kann, ohne dass jemand wartet, ohne dass man jemanden zu kurz kommen lässt, ist es natürlich anders bei Müttern, die ihre Kinder zu Hause haben. (...) Ich glaube (...) nicht, dass sie insgesamt das gleiche Pensum schaffen können wie jemand, der halt die Möglichkeit hat, auch mal abends um neun oder zehn hier zu sein" (Abteilungsleiterin Produktmanagement).

Die zentrale Argumentationsfigur, reduzierte Arbeitszeiten in leitenden Positionen abzulehnen, bezieht sich grundlegend auf das Führungsverständnis der Personalverantwortlichen. Es zeigen sich zwei grundlegende Argumentationslinien: Erstens wird eine immerwährende Ansprechbarkeit für die Mitarbeiter als Bedingung erfolgreicher Führung postuliert und mit der Vorbildfunktion des Vorgesetzten im Umgang mit der Arbeitszeit verknüpft. Dahinter verbirgt sich zugleich die Auffassung, dass Führungspositionen nur in Vollzeit uneingeschränkte Kontrollmöglichkeit gewähren. Reduzierte Arbeitszeiten werden in diesem Kontext auch implizit mit einer laxen Arbeitsmoral gleichgesetzt und somit negativ beurteilt. Zweitens werden geteilte Leitungspositionen aufgrund von unterschiedlichen Führungsstilen von der Mehrheit der Befragten als ineffizient beurteilt. Die Teilung eines „homogenen Arbeitsguts" sei nicht möglich (Hauptabteilungsleiter Personal). Personalpolitische Möglichkeiten bei der Reduzierung von Arbeitszeiten in Führungspositionen, wie die Delegation von Fachoder Führungsaufgaben durch Stellvertreterregelungen, die zeitliche oder inhaltliche Teilung von Arbeitsplätzen sowie arbeitsorganisatorische Möglichkeiten der flexiblen Erreichbarkeit, werden von den befragten Personalverantwortlichen nicht erwogen. Dies zeigt beispielhaft die Stellungnahme einer Personalverantwortlichen zu reduzierten Arbeitszeiten mit der Hälfte der Arbeitszeit auf der untersten Führungsebene.
„Ich weiß nicht, wie das gehen soll, (...) die Gruppenleiter sind Ansprechpartner für ihre Mitarbeiter, wenn Probleme auftreten, Rückfragen und, und, und, und wenn die Mitarbeiter jetzt sitzen und arbeiten und die Gruppenleiterin ist um $12.00 \mathrm{Uhr}$ nach Hause gegangen, wen sollen die denn fragen, es ist keiner mehr da zu fragen, das kann es nicht sein" (Abteilungsleiterin Controlling).

\subsection{NEGATIVE KONNOTATIONEN VON TEILZEITARBEIT UND GESCHLECHTERKONSTRUKTIONEN}

Wird der Vollzeitstandard in Verbindung mit höchsten Anforderungen an die zeitliche Verfügbarkeit als Voraussetzung für die Ausübung von Führungspositionen postuliert, so bildet er gleichzeitig den Maßstab für eine negative Bewertung von Teilzeitarbeit. Dabei dominiert in den Haltungen der befragten Führungskräfte das Verständnis, Arbeitszeitreduzierung in Führungspositionen als berufliche Einschränkung und geringeres betriebliches Engagement zu interpretieren. In der Perspektive der Befragten werden reduzierte Arbeitszeiten mit geringerer Arbeitsqualität gleichgesetzt, die den Ausschluss aus dem Segment der Führungspositionen legitimiert.

„Aus meiner Sicht ist es mehr die Frage von, sagen wir mal, unterschiedlichen Prioritäten in den Lebensphasen. Dass dann wichtig ist, dass die Firma einem zur Seite steht, wenn man sich fürs Kind entscheidet. Eine andere Frage ist, ob ich von der Firma erwarten kann, dass sie mich karrieremäßig fördert, trotz der Teilzeit und trotzdem es andere Mitarbeiter gibt, die $100 \%$ geben können. (...) Und dass man dann natürlich als Firma auch versucht, das Maximale oder die beste Besetzung zu finden, ist dann auch klar" (Hauptabteilungsleiter Marketing).

Als Voraussetzung für den weiteren Aufstieg in eine höhere Führungsposition wird die Entscheidung "gegen die Familie“ postuliert.

„Natürlich hätte es im Laufe der Jahre mindestens drei Kolleginnen gegeben, die auf dem gleichen intellektuellen Ausstattungslevel mit den gleichen Managementqualifikationen und so weiter und so weiter diese Jobs auch hätten bekleiden können. Und wären wir sozusagen biologisch äquivalent, dann wären drei dieser Directors heute Frauen. (...) Ich habe eigentlich jetzt nur eine Kollegin im Nachwuchsbereich, die sicherlich auch in absehbarer Zeit eine dieser
Direktorenjobs bekleiden wird, die sich aber ganz klar gegen die Familie und für den Berufentschieden hat" (Bereichsleiter Marketing).

In den Deutungen der Personalverantwortlichen werden in Verbindung mit reduzierten Arbeitszeiten Geschlechterkonstruktionen deutlich, die wiederum mit der Perspektive der Unvereinbarkeit der Lebensbereiche von Berufsarbeit und Familie verknüpft sind. Das Geschlechtermodell, das hier zum Ausdruck kommt, offenbart eine klassische Geschlechterkonstruktion, nämlich jene, dass Männer Sorgearbeiten per Definition nicht übernehmen, verbunden mit der Normierung, dass sie diese auch nicht zu übernehmen haben. Im Kontrast dazu wird Fürsorgearbeit für die Erwerbsarbeit negativ konnotiert und mit einer weiblichen Geschlechterkonstruktion in Beziehung gesetzt.

\subsection{ZWISCHENFAZIT}

Unsere Untersuchungsergebnisse, die hier nur auszugsweise vorgestellt werden können, zeigen, dass Führungskräfte reduzierte Arbeitszeiten in Führungspositionen generell ablehnen. Ebenso zurückgewiesen wird der berufliche Aufstieg von hoch qualifizierten Teilzeitbeschäftigten mit Kindern in leitende Positionen wie auch ein weiterer Aufstieg innerhalb des Führungskräftebereichs. Zum einen machen die Argumentationsmuster, die von den befragten Personalverantwortlichen herangezogen werden, darauf aufmerksam, dass von einer objektiven, nicht auf den Einzelfall bezogenen Ungeeignetheit einer Führungsposition für Arbeitszeitreduzierungen ausgegangen wird. Zum anderen fließen normative Haltungen in die Ablehnung der Teilzeitrechte ein, die nicht als sachliche Ablehnungsgründe gelten können. Die dominanten Interpretationen der Personalverantwortlichen erweisen sich vor dem Hintergrund der gesetzlichen Ablehnungsgründe als unzulässig. Das Ziel des Gesetzgebers, kürzere Arbeitszeiten mit einem Rechtsanspruch auch auf den höchsten Ebenen der betrieblichen Hierarchie zu fördern, wird konterkariert. 


\section{Handlungslogiken im Umgang mit Teilzeitrechten}

In unseren Analysen war es der Regelfall, dass die befragten Personalverantwortlichen ihre Perspektiven und Entscheidungen verbal mit einem betrieblichen Effizienzkalkül begründen. Gleichzeitig werden normative Haltungen deutlich, die mit den vorgetragenen Argumentationsmustern nicht korrespondieren. Woraus resultiert nun also die starke Ablehnung der gesetzlichen Arbeitszeitrechte und die damit verbundene Veränderung des dominanten Vollzeitstandards mit entgrenzten Arbeitszeiten? Wie erklärt sich die negative Bewertung reduzierter Arbeitszeiten, warum werden in diesem Zusammenhang immer wieder Geschlechterstereotypisierungen ins Spiel gebracht?

In einem weiteren Forschungsschritt wurde daher der Frage nachgegangen, welchen Logiken das Handeln der betrieblichen Akteure folgt, d.h., woraus der restriktive Umgang mit den Teilzeitrechten resultiert. Dazu musste sich unser Interpretationsblick verändern, da es zu erklären galt, wie sich die Deutungen und Perspektiven der Personalverantwortlichen konstituierten, welche Handlungsmuster und Erfahrungen ihnen zugrunde lagen und in welchem Maße betriebliche Bedingungen ihr Handeln beeinflussten. Methodisch wurden bei diesem Forschungsschritt rekonstruktive Fallanalysen vorgenommen. Deren Ziel ist es, die Regeln zu ergründen, nach denen sich ein Fall generiert. Das Interesse richtet sich damit auf grundlegende Wirkungszusammenhänge, die am konkreten Einzelfall untersucht werden und zu einer Typenbildung herangezogen werden können (Rosenthal 2005, S. 56ff.; Bude 2003, S. 60f.; Apitzsch 2003). ${ }^{7}$ Im Folgenden werden die Ergebnisse der zwei durchgeführten Fallanalysen zu den Interviews mit den Personalverantwortlichen zunächst fallübergreifend, dann im Einzelnen vorgestellt.

\subsection{FALLÜBERGREIFENDE ERGEBNISSE}

Fallübergreifend zeigte sich, dass die restriktive und selektive Praxis im Umgang mit den gesetzlichen Teilzeitrechten im Segment hoch qualifizierter Beschäftigung maßgeblich bestimmt ist von der lebensge- schichtlichen Dimension der betrieblichen Funktionsträger und -trägerinnen, d.h. von den Bedingungen, Erfahrungen und Deutungsmustern der eigenen Lebensführung im Umgang mit hoch qualifizierter Berufsund Fürsorgearbeit in Verbindung mit dem jeweiligen Organisationskontext. Nicht ökonomische Effizienzkalküle sowie betriebliche Organisationsstrukturen, die in den Deutungen der Befragten im Vordergrund für ihr Handeln stehen, verhindern eine Umsetzung von reduzierten Arbeitszeiten. Es zeigt sich vielmehr der grundlegende Wirkungszusammenhang, dass eine Umsetzung der gesetzlichen Normierungen dort nicht erfolgt, wo eigene - kulturell dominante - Arbeits- und Lebensmodelle, die von einer Unvereinbarkeit von hoch qualifizierter Berufs- und Fürsorgearbeit bestimmt sind, den gesetzlichen Normen gegenüberstehen.

Zentrale Unterschiede bestehen dennoch darin, wie es zu dieser Bestätigung des eigenen Arbeits- und Lebensmodells mit entgrenzten Arbeitszeiten kommt. Es lassen sich hierbei verschiedene Handlungstypen differenzieren. Zunächst wird ein Typus vorgestellt, der Fürsorgearbeit in einem Arbeits- und Lebensmodell in Managementpositionen grundlegend ausschließt unter Verweis auf die Notwendigkeit betrieblicher Allzeitverfügbarkeit (Abschnitt 4.2). Anders konstituiert sich das Handlungsmuster eines Typus, der in der betrieblichen Praxis selbst keine Möglichkeit erhalten hat, hoch qualifizierte Berufs- und Fürsorgearbeit in einem Modell mit reduzierten Arbeitszeiten in Führungspositionen zu verbinden (Abschnitt 4.3). ${ }^{8}$

\subsection{ARBEIT ALS LEBENSFORM - BETRIEBLICHE ALLZEITVERFÜG- BARKEIT UND MÄNNLICHE IDENTITÄTSKONSTRUKTION}

Herr S. repräsentiert diesen Typus. Er ist Mitte 50, Personalverantwortlicher im oberen Management und arbeitete während seiner gesamten Berufslaufbahn Vollzeit. Herr S. lebt ein Arbeits- und Lebensmodell, das von einer strikten Trennung der Bereiche von hoch qualifizierter Berufsarbeit und Fürsorgearbeit geprägt ist. Die zwei mittlerweile erwachsenen Töchter hat seine Frau betreut, die ihre Erwerbstätigkeit als Akademikerin nach der Geburt der Töchter aufgegeben hat. Seine Perspektive auf hoch qualifizierte Berufsarbeit im Unternehmen ist davon bestimmt, sie mit der
Anforderung einer entgrenzten Arbeitszeit zu verknüpfen, die in einer Weise definiert ist, dass sie die Gestaltung fürsorglicher Beziehungen nicht zulässt.

Als grundlegendes Handlungsmuster in der betrieblichen Praxis wird erkennbar, dass es zu einem Umgang mit den Teilzeitrechten kommt, der durch Nichtumsetzung von Teilzeitansprüchen in Führungspositionen, Marginalisierung der weiblichen Beschäftigten (Ausstattung mit unzureichenden Ressourcen), Dequalifizierung (Zuweisung qualifikationsinadäquater Arbeitsplätze) bis hin zur Ausgrenzung aus dem Unternehmen gekennzeichnet ist. Zentral ist dabei, dass Herr S. die eigene Verantwortlichkeit als Personalverantwortlicher für die Zuweisung der Arbeitsplätze und die Gestaltung der Arbeitsbedingungen der zeitreduziert arbeitenden Mitarbeiterinnen ausblendet. Außen vor bleibt in seiner Darstellung auch, dass er aufgrund der eigenen personalpolitischen Entscheidungen, im Fall der Schwangerschaft seiner Mitarbeiterinnen unbefristet Vollzeitarbeitskräfte auf deren Arbeitsplätzen einzustellen, „überschüssiges“ Teilzeitpersonal reduzieren muss, um die für seinen Arbeitsbereich geltenden Personalvorgaben zu erfüllen.

Demgegenüber steht in seiner Präsentation zum einen der Rekurs auf das Deutungsmuster der ökonomisch bestimmten Rationalität seines Handelns in Form eines zwingend für das Organisationsüberleben notwendigen entgrenzten Arbeitszeitstandards. Zum anderen greift Herr S. auf das

\footnotetext{
7 Hierbei ist wichtig zu beachten, dass im Rahmen der Grundannahmen interpretativer Sozialforschung eine Verallgemeinerung der Ergebnisse in anderer Form vorgenommen wird als bei quantitativen Analysen. Die Typenbildung wird verstanden als eine Abstraktion der Fallstruktur (WohlrabSahr 1994, S. 273). Geschlossen wird daher beim Typus auf alle gleichartigen Fälle, d.h. auf Fälle, die einem ähnlichen Regelsystem folgen (Rosenthal 2005, S. 75). Die für die Auswertung herangezogenen Fälle wurden nach den Regeln des maximalen Kontrasts ausgewählt. Die Grundlage der Fallauswahl bildete dabei die grundlegend unterschiedliche Perspektive auf die Vereinbarkeit von Familie und Beruf der Befragten.

8 Bei rekonstruktiven Fallanalysen handelt es sich um sehr komplexe, systematisch kontrollierte Auswertungen sprachlichen Datenmaterials. An dieser Stelle werden die Ergebnisse platzbedingt in ergebnisorientierter Kurzform und unter Verzicht auf die Präsentation von Interviewmaterial dargelegt. Zu den ausführlichen Darstellungen der beiden Fälle siehe Koch (2007, S. 77-135).
} 
Deutungsmuster einer scheinbar natürlichen Geschlechterdifferenz zurück. Damit deutet er die Marginalisierung und Ausgrenzung der weiblichen Beschäftigten als Selbstausschluss aufgrund einer für den Betrieb dysfunktionalen Familienorientierung und etabliert im Gegenzug eine männliche Geschlechterkonstruktion für die betriebliche Praxis, die den Ausschluss von Fürsorgearbeit beinhaltet. Über beide Deutungsmuster kommt es somit zu einer Delegitimierung der gesetzlichen Teilzeitrechte für hoch qualifizierte Berufsarbeit. Bezogen auf die Organisationsebene zeigt sich, dass die Handlungspraxis von Herrn S. im Umgang mit den Teilzeitbeschäftigten durch das Unternehmen gestützt wird.

Mit den Deutungen der Dichotomie von hoch qualifizierter Berufs- und Fürsorgearbeit, die die eigene betriebliche Handlungspraxis als Personalverantwortlicher legitimieren, korrespondiert der Blick auf das eigene Arbeits- und Lebensmodell. Dieses wird als alternativlos unter der Prämisse eines beruflichen Aufstiegs in eine Führungsposition interpretiert und mit der Figur der Unvereinbarkeit von hoch qualifizierter Berufsarbeit und Fürsorgearbeit verbunden. Der Stellenwert der Fürsorgearbeit ist hierarchisiert, indem diese als Behinderung hoch qualifizierter Berufsarbeit definiert wird. In der eigenen Arbeitsidentität werden Leistungsfähigkeit und Qualifikation über die Realisierung unbegrenzter Zeitverfügbarkeit miteinander verwoben.

Die Perspektive der Unvereinbarkeit hoch qualifizierter Berufs- und Fürsorgearbeit wird aufrechterhalten, obgleich Herr S. sich lebensgeschichtlich mit den Konsequenzen seines Arbeits- und Lebensmodells konfrontiert sieht, als seine Töchter erwachsen sind und er die fehlende Beziehung als emotionalen Verlust erfährt. Diese Erfahrung integriert er nicht in die Perspektive auf das eigene Arbeits- und Lebensmodell, es wird weiterhin als das einzig richtige legitimiert. Herr S. überträgt das eigene Arbeits- und Lebensmodell mit dem Postulat einer strikten Trennung von hoch qualifizierter Berufs- und Fürsorgearbeit auf sein Handeln als Personalverantwortlicher im Unternehmen. Die gewählte Perspektive erhält die Funktion, den eigenen Arbeits- und Lebensentwurf zu bestätigen und dessen Infragestellung durch den Verlust familiärer Bindungen außen vor zu halten. Das eigene Arbeits- und Lebensmodell und die damit verbundenen
Kosten, der Ausschluss von Fürsorgearbeit, werden legitimiert.

Generalisierend: Der Repräsentant dieses Typus realisiert ein Arbeits- und Lebensmodell mit entgrenzten Arbeitszeiten. Fürsorgearbeit ist in der eigenen Perspektive nicht Teil des Lebensentwurfs. Im Betrieb gestaltet er die Unvereinbarkeit von Erwerbs- und Fürsorgearbeit. Reduzierte Arbeitszeiten in Führungspositionen werden nicht umgesetzt, weil das eigene Arbeits- und Lebensmodell und der damit verbundene berufliche Erfolg nicht infrage gestellt werden sollen. Darüber kommt es zu einer Delegitimierung der Teilzeitrechte. Dies kann in einem Organisationskontext geschehen, der die Vorgehensweise des Personalverantwortlichen stützt.

\subsection{FÜRSORGE ZWISCHEN WÜNSCHEN NACH ANERKENNUNG UND HEGEMONIALEN ARBEITSZEIT- MODELLEN}

Frau B. steht für diesen Typus. Sie ist promovierte Naturwissenschaftlerin, Anfang 40 und arbeitet als Führungsverantwortliche im mittleren Management eines großen Unternehmens in Vollzeit. Ihre zwei kleinen Kinder werden vornehmlich von einer Kinderfrau und ihrem Mann betreut, der ebenfalls in Vollzeit tätig ist. Frau B. praktiziert seit Jahren, sowohl vor ihrer Beschäftigung als Personalverantwortliche als auch in der jetzigen Führungsposition, ein Arbeitsmodell mit entgrenzten Arbeitszeiten während der Arbeitswoche. Fürsorgearbeit leistet sie am Wochenende und in der Nacht. Charakteristisch für ihre Perspektive ist es, Fürsorgearbeit in der Kombination mit hoch qualifizierter Berufsarbeit anerkennen zu wollen.

Ihr zentrales Umgangsmuster als Personalverantwortliche ist dennoch von einer Abwertung von Teilzeitarbeitsmodellen im Vergleich zu Vollzeitarbeitsmodellen bei Fürsorgearbeit gekennzeichnet. Dies geschieht zum einen dadurch, dass sie den Teilzeitstatus der Mitarbeiterinnen ihres Arbeitsbereichs über die Zuweisung geringerwertiger Arbeitsanforderungen marginalisiert. Zum anderen werden reduzierte Arbeitszeiten in Führungspositionen negativ konnotiert.

Diese Hierarchisierung wird über verschiedene Deutungsmuster gestützt: Zum einen geschieht dies durch die Interpretation des Teilzeitstatus als Schutzraum. Zum anderen wird über den Topos der geringe- ren Leistungsfähigkeit von Beschäftigten mit reduzierten Arbeitszeiten im Vergleich $\mathrm{zu}$ einem Arbeitsmodell mit entgrenzten Arbeitszeiten die Gleichsetzung von Arbeitsqualität mit Arbeitsquantität transportiert und das Verhalten des Unternehmens als ökonomisch irrational konnotiert, wenn es Modelle mit reduzierten Arbeitszeiten in Führungspositionen zulässt. Schließlich ermöglicht der Rekurs auf eine weibliche Geschlechterkonstruktion, vermehrte Fürsorgearbeit als Hindernis für den Aufstiegswillen der weiblichen Beschäftigten zu deuten und die geringere Repräsentanz von Frauen in Führungspositionen als Selbstausschluss zu legitimieren. Über diese Deutungsmuster kommt es im Ergebnis zu einer Ausblendung und Delegitimierung der Teilzeitrechte als kollektivem Geltungsanspruch, um hoch qualifizierte Berufs- und Fürsorgearbeit kombinieren zu können.

Über die Betrachtung der lebensgeschichtlichen Dimension erklären sich die Umgangsmuster von Frau B., die sich auf die Gewährung von Teilzeitmodellen und die Teilzeitrechte beziehen, und ihre damit verknüpften Deutungsmuster: Die Schilderungen zur eigenen Erwerbsbiografie zeigen, dass der Wahl ihres Arbeitsmodells als latente Struktur die Wahrnehmung einer Gefährdung des eigenen beruflichen Status zugrunde liegt, aufgrund von Mutterschaft nicht mehr als gleichwertig und damit konkurrenzfähig angesehen zu werden. Frau B. wählt mit ihrer Form, hoch qualifizierte Erwerbstätigkeit und Familie zu kombinieren, ein Modell, das sich in seiner Funktionalität für die Organisation nicht von hoch qualifizierten Führungskräften, die keine Fürsorgearbeit leisten, unterscheidet. Sie modifiziert das Modell dennoch entscheidend, indem sie Zeiträume der Betreuungsarbeit für ihre Kinder reserviert.

In der Fallrekonstruktion wird deutlich, dass ihr Wunsch, als Führungsverantwortliche nach der Geburt ihres zweiten Kindes Teilzeit zu arbeiten, ebenso wie der Wunsch ihres Mannes, zeitreduziert zu arbeiten, in den jeweiligen Unternehmen abgelehnt wurde. Im Ergebnis resultieren die Hierarchisierung von Teilzeitarbeitsmodellen, die mit Fürsorgearbeit verbunden werden, und die Ablehnung dieser Modelle für den Bereich hoch qualifizierter Berufsarbeit mit Leitungsverantwortung aus der Notwendigkeit, das eigene Arbeits- und Lebensmodell in seiner jetzigen Form und den beabsichtigten weiteren beruflichen 
Aufstieg zu schützen. Das eigene gegenwärtig praktizierte Arbeits- und Lebensmodell wird als Erfolgsmodell präsentiert unter Ausschluss anderer, vormals biografisch relevanter anderer Optionen. Die eigene Anpassungsleistung führt dazu, dass hoch qualifizierte Berufsarbeit und Fürsorgearbeit in Kombination mit Modellen reduzierter Arbeitszeiten über die Kodierung geringerer Leistungsorientierung abgewertet werden. Die neuen Teilzeitrechte können somit nicht als Instrument einer Veränderung der vorherrschenden Arbeitszeitkultur genutzt werden.

Generalisierend: Die Repräsentantin steht für einen Typus, den eine grundlegende Ambivalenz kennzeichnet zwischen dem Wunsch nach Anerkennung von Fürsorgearbeit und einer beruflichen Praxis mit entgrenzten Arbeitszeiten und dadurch stark eingeschränkten Möglichkeiten, Fürsorgearbeit zu realisieren. Das hegemoniale Arbeits- und Lebensmodell mit entgrenzten Arbeitszeiten wird modifiziert, indem Zeit für Fürsorgearbeit gegen das Postulat uneingeschränkter Verfügbarkeit und trotz entgrenzter Arbeitszeiten ,verteidigt“ wird. Die eigene Grundhaltung ist davon bestimmt, Fürsorgearbeit als wünschens- und unterstützenswert zu betrachten.

Die negative berufliche Erfahrung, dass reduzierte Arbeitszeiten in einer Führungsposition vorenthalten wurden, wird allerdings in die Anforderung an hoch qualifizierte Beschäftigte transformiert, in Führungspositionen ein Arbeits- und Lebensmodell mit entgrenzten Arbeitszeiten zu übernehmen. Ein positiver Bezug auf Fürsorge in Kombination mit hoch qualifizierter Berufsarbeit kann zwar als Teil eines kulturellen Musters der Anerkennung von Fürsorgearbeit entfaltet werden, wird jedoch für die berufliche Praxis als nicht realisierbar gedeutet.

\section{Fazit und Ausblick}

Wie die Forschungsergebnisse zeigen, erweist sich die Voraussetzung entgrenzter
Arbeitszeiten für Führungspositionen und die damit verbundene pauschale Ablehnung der Rechtsansprüche als zentrale Problemkonstellation. Das dafür grundlegend herangezogene Begründungsmuster ökonomischer Effizienz wird dabei von den Befragten in solcher Form eingesetzt, dass die Gewährung von Arbeitszeitreduzierungen im Führungskräftebereich im Ergebnis ausgeschlossen ist. Dieser Mechanismus verhindert die Umsetzung der gesetzlichen Normen.

Die Ergebnisse der Fallanalysen vermögen weitergehend zu erklären, wie sich eine solche Perspektive entwickeln kann und welche Erfahrungen sich damit verknüpfen. Die Analysen geben Einblick in einen komplexen Wirkungszusammenhang: Der Rückgriff auf den Diskurs ökonomischer Effizienz als Begründungsmuster der Teilzeitablehnung (wie auch der Bezug auf Geschlechterstereotypien) erweist sich als Legitimationsstrategie, das eigene Arbeitsund Lebensmodell mit entgrenzten Arbeitszeiten zu schützen. Die individuellen biografischen Erfahrungen der betrieblichen Akteure mit hoch qualifizierter Berufsarbeit werden - gestützt durch den jeweiligen Organisationskontext - auf die betriebliche Handlungspraxis im Umgang mit den Teilzeitrechten übertragen. Die Ergebnisse machen darauf aufmerksam, dass die neuen Arbeitszeitrechte für einen anderen Arbeits- und Lebensentwurf der Kombination von Erwerbs- und Fürsorgearbeit und damit im Spannungsfeld zwischen Anerkennung und Abwehr von Fürsorgewünschen stehen. Sie lassen sich somit verstehen als über rechtliche Normierung politisierte, vormals als privat definierte soziale Bedürfnisse nach Fürsorge.

Für die betriebliche Zeitpolitik und die Arbeit betrieblicher Interessenvertretungen bieten sich hier wichtige Chancen, diese „lebensweltlichen“ Interessen der Beschäftigten aufzugreifen und mit dazu beizutragen, eine betriebliche Kultur gleichwertiger Arbeits- und Lebensentwürfe zu gestalten, die es leistet, Fürsorgearbeit zu integrieren. ${ }^{9}$

Die Untersuchungsergebnisse verweisen nicht zuletzt auf die Notwendigkeit weiterer Maßnahmen. Dies sind einerseits Initiativen in den Unternehmen, alternative Arbeitszeitmodelle in der Umsetzung reduzierter Arbeitszeiten zu entwickeln. Andererseits erscheinen weitere gesetzliche Maßnahmen notwendig, sollen gesellschaftliche Veränderungen nicht ausschließlich den individuellen Lernprozessen Einzelner überantwortet werden. Hierzu gehören neben einer effektiveren Gestaltung der Teilzeitrechte ${ }^{10}$ nach wie vor gesetzliche Regelungen, die zu einer stärkeren Inanspruchnahme von Erziehungszeiten durch Väter führen, insbesondere durch individuelle Ansprüche auf Elternzeit mit jeweils gleichem Zeitumfang. Ebenso wichtig ist weiterhin die noch ausstehende arbeitsrechtliche Gleichstellungsgesetzgebung in der Privatwirtschaft.

Gesellschaftspolitisch aktuell ist unter dem Signum der Geschlechtergleichheit schließlich noch immer die Umverteilung des gesellschaftlich erbrachten Arbeitsvolumens. Dies erfordert einen Paradigmenwechsel hin zu einer Neudefinition des Normalarbeitsverhältnisses über einen kürzeren Vollzeitstandard, der mit der ausreichenden Bereitstellung öffentlicher Infrastruktur für Kinderbetreuung und Maßnahmen der Gleichverteilung von Erwerbsund von Pflege- bzw. Betreuungsarbeit zwischen den Geschlechtern kombiniert werden kann.

\footnotetext{
Vgl. hierzu auch das Plädoyer von Jürgens für eine kritische Arbeitsforschung, für die Jürgens einfordert, die Ressourcen, Bedingungen und Konstellationen zu identifizieren, die die Subjekte in der Verteidigung ihrer individuellen und lebensweltlichen Interessen unterstützen (Jürgens 2006, S. 282).

10 Das betrifft die Vereinheitlichung und Spezifizierung der Ablehnungsgründe in den beiden Gesetzesgrundlagen. Dies könnte mit einem wirksamen Durchsetzungsmechanismus verknüpft werden, um Diskriminierungen abzubauen. In Analogie zum Kündigungsschutz im Mutterschutz und in der Elternzeit ließe sich hier über die Schaffung einer überbetrieblichen Stelle nachdenken, die die Ablehnung des Teilzeitverlangens zu genehmigen hat.
} 
Apitzsch, U. (2003): Biographieforschung, in: Orth, B. et al., (Hrsg.): Soziologische Forschung: Stand und Perspektiven, Opladen, S. 95-110 Bäcker, G./Koch, A./Vornmoor, A. (2007): Chancengleichheitsorientierte Arbeitszeitpolitik in der betrieblichen Praxis - Eine Wirkungsanalyse des Bundeserziehungsgeldgesetzes und des Teilzeit- und Befristungsgesetzes, Projektbericht, Düsseldorf

Bäcker, G./Stolz-Willig, B. (1993): Geschlechterrollen und sozialpolitische Umorientierung, in: Gewerkschaftliche Monatshefte 7, S. 414-432 BErzGG (2001): Gesetz zum Erziehungsgeld und zur Elternzeit (Bundeserziehungsgeldgesetz), Fassung der Bekanntmachung vom 12.12. 2001, BGBI. I, S. 3359

Bothfeld, S. (2005): Vom Erziehungsurlaub zur Elternzeit - Politisches Lernen im Reformprozess, Frankfurt a. Main

Bude, H. (2003): Fallrekonstruktion, in: Bohnsack, R. et al. (Hrsg.): Hauptbegriffe qualitativer Sozialforschung. Ein Wörterbuch, Opladen, S. 60-61

Buschmann, R./Dieball, H./Stevens-Bartol, E. (2001): Das Recht der Teilzeitarbeit - Kommentar für die Praxis, Frankfurt a. Main

Holst, E. (2006): Führungspositionen - Frauen geringer entlohnt und nach wie vor seltener vertreten, in: DIW-Wochenbericht 25, S. 365-371 Jürgens, K. (2006): Arbeits- und Lebenskraft - Reproduktion als eigensinnige Grenzziehung, Wiesbaden

Klammer, U./Daly, M. (2003): Die Beteiligung von Frauen an europäischen Arbeitsmärkten, in: Gerhard, U. et al. (Hrsg.): Erwerbstätige Mütter - Ein europäischer Vergleich, München, S. 193-214

Kleinert, C./Kohaut, S./Brader, S./Lewerenz, J. (2007): Frauen an der Spitze - Arbeitsbedingungen und Lebenslagen weiblicher Führungskräfte, Frankfurt a. Main
Koch, A. (2001): Neubewertung der Familienarbeit in der Sozialpolitik? Zur Reform von Erziehungsgeld und Erziehungsurlaub und alternativen Konzeptionen, in: Feministische Studien 1, S. 48-61

Koch, A. (2007): Fallauswertungen, in: Bäcker, G./Koch, A./Vornmoor, A.: Chancengleichheitsorientierte Arbeitszeitpolitik in der betrieblichen Praxis - Eine Wirkungsanalyse des Bundeserziehungsgeldgesetzes und des Teilzeit- und Befristungsgesetzes, Düsseldorf, S. 77-135

Kurz-Scherf, I. (1987): Zeit(t)räume per Tarifvertrag Oder: Die Renaissance der betriebsnahen Tarifpolitik, in: WSI-Mitteilungen 8, S. 492-502 Perreng, M./Nollert-Borasio, C. (2006): Allgemeines Gleichbehandlungsgesetz - Basiskommentar zu den arbeitsrechtlichen Regelungen, Frankfurt a. Main

Pfarr, H. (1994): Welche Maßnahmen empfehlen sich, um die Vereinbarkeit von Berufstätigkeit und Familie zu verbessern?, in: Zeitschrift für Rechtspolitik 8, S. 309-314

Rosenthal, G. (2005): Interpretative Sozialforschung - Eine Einführung, Weinheim

TzBfG (2001): Gesetz über Teilzeitarbeit und befristete Arbeitsverträge und zur Änderung und Aufhebung arbeitsrechtlicher Bestimmungen vom 21. Dezember 2000, BGBI, I, Nr. 59, 1966-1970 Wohlrab-Sahr, M. (1994): Vom Fall zum Typus, in: Diezinger, A. et al. (Hrsg.): Erfahrung mit Methode, Freiburg i.Br., S. 269-299

Weinkopf, C./Kalina, T. (2006): Mindestens sechs Millionen Niedriglohnbeschäftigte in Deutschland: Welche Rollen spielen Teilzeitbeschäftigte und Mini-Jobs, IAT-Report 3, Gelsenkirchen 